in den Formeln ( 1 ) bis (4) mit dem Index 0 bezeichneten Grössen auf den einfallenden Strahl, die mit dem Index $i$ bezeichneten auf den austretenden Strahl bezogen und seien

$$
\begin{gathered}
X_{0}=\frac{\mathbf{1}-\mathfrak{A}_{i}}{\mu_{0} \mathfrak{B}_{i}} \text { und } \quad X_{i}=\frac{\mathbf{1}-\mathfrak{D}_{i}-k_{i}}{\mu_{i} \mathfrak{B}_{i}} \\
\text { wobei } \quad k_{i}=\left(B_{i} g_{i}-A_{i} h_{i}\right)+\left(\Gamma_{i} f_{i}-\Delta_{i} e_{i}\right)+\left(f_{i} g_{i}-e_{i} h_{i}\right)
\end{gathered}
$$

ist, die Entfernungen der Knotenpunkte von dem Mittelpunkte der ersten, bezw. letzten brechenden Fläche, und

$$
\begin{aligned}
& \boldsymbol{\Phi}_{0}=\frac{\mathrm{I}}{\mu_{0} \mathfrak{B}_{i}} \\
& \boldsymbol{\Phi}_{i}=\frac{1-k_{i}}{\mu_{i} \mathfrak{B}_{i}}
\end{aligned}
$$

die Entfernungen der Brennebenen von den Knotenpunkten für die parallel austretenden bezw. parallel einfallenden Strahlen. Sind $W_{0}, W_{i}$ die Entfernungen von Gegenstand und Bild von den Knotenpunkten, so ist

$$
\frac{\Phi_{i}}{W_{i}}+\frac{\Phi_{0}}{W_{0}}==\mathbf{I}
$$

$\mathrm{Da}$ die Ausdrücke für $\mathfrak{A}_{i}, \mathfrak{V}_{i}, \mathfrak{\Xi}_{i}, \mathfrak{D}_{i}$ und $k_{i}$ von der Linsenöffnung und dem Bildwinkel abhängig sind, überdiess aber auch von den Brechungsexponeaten der verschiedenen Medien, so wird die Lage der Knotenpunkte und Brennebenen verschieden für die Randstrahlen und Centralstrahlen, verschieden je nach der Lage des Objectes in oder ausserhalb der Axe und verschieden für verschiedenfarbiges Licht. Man sieht aber sofort, dass, wenn man zunächst $A_{i}, B_{i}, A_{i}$ so bestimmt, dass es für verschiedene Strahlengattungen denselben Werth erlangt, Knotenpunkte und Brennebenen für die Centralstrahlen für verschiedene Farben iden. tisch werden, daher die chromatische Abweichung und Verschiedenheit der Farbenvergrösserung weggeschafft sind. Macht man dann weiter $e_{i}=f_{i}=g_{i}=h_{i}=0$, so ist die sphärische Abweichung in und ausser der Axe, d. h. sphärische Aberration, Astigmatismus, Coma und Bildkrümmung weggeschafft. Mit Rücksicht auf die zweiten Potenzen von Linsenöffnung und Bildwinkel wird nun die sphärische Aberration in der Axe für die ganze Linsenöffnung,

Heidelberg 1898 Juli 13. d. $h$. für Strahlen in jeder beliebigen Entfernung von der Axe gleichzeitig beseitigt, eine durch die numerischen Rechnungen schon lange bekannte Thatsache: die Beseitigung der sphäri. schen Aberration für die Randstrahlen beseitigt genähert auch die sphärische Aberration für die übrigen Strahlen. Nicht dasselbe gilt aber (nicht nur bei den höheren, sondern schon mit Rücksicht auf die zweiten Potenzen von Oeffnungsund Bildwinkel) für die Strahlen ausser der Axe, d. h. Astigmatismus, Coma und Bildkrümmung können nicht in dem. selben Maasse weggeschafft werden.

Die Zahl der zu erfuillenden Bedingungsgleichungen für gegebene Vorschriften ergeben sich hieraus leicht: für die Elimination der chromatischen Aberration und Verschiedenheit der Farbenvergrösserung ergeben sich für zwei Farben drei Bedingungen, für jede weitere Farbe wieder drei Bedingungen; Wegschaffung der sphärischen Abweichung, der Coma und der Bildkriummung für jede Farbe, jeden Oeffnungsund jeden Bildwinkel je vier Bedingungen. Also z. B. für die Vereinigung zweier Farben in der Axe und Berücksichtigung eines einzigen Oeffnungs- und eines einzigen Bildwinkels sieben Bedingungen, wozu noch die gegebene Brennweite als achte Bedingung tritt.*) Hieraus ist ersichtlich, dass die grosse Mehrzahl der jetzt construirten Linsensysteme das angestrebte Ziel mit einem ganz bedeutenden und unnöthigen Aufwand von brechenden Flächen erreicht, und dieses bei der Construction zukünftiger Linsensysteme sowohl im Interesse der Einfachheit als der Preise der Linsensysteme zu berücksichtigen sein wird. Allerdings wächst die Zahl der Bedingungsgleichungen ausserordentlich rasch, sobald die Anforderungen erhöht ${ }^{-}$werden. Soll z. B. das System für drei Farben, zwei verschiedene Oeffnungen und zwei Bildwinkel fehlerfrei sein, so wird die Zahl der Bedingungsgleichungen 50. Für gegebene Linsensysteme wird man daher eine Reihe von Bedingungsgleichungen auszuwählen haben, welche dem angestrebten $Z$ wecke am besten entsprechen.

\section{N. Herz.}

*) Dass überdiess das Verhältniss zwischen Oeffnung, Krümmung und Linsendicke kein unmögliches sein darf, braucht nicht erst besonders hervorgehoben $\mathrm{zu}$ werden.

\title{
Beobachtung der Mondfinsterniss 1898 Juli 3
}

\author{
auf der Universitäts-Sternwarte zu Charkow.
}

Während der fast totalen Mondfinsterniss gelang hier die Beobachtung der Verfinsterung einer Anzahl von Kratern. Der verfinsterte Theil des Mondes erschien ziemlich dunkel, doch konnten im 6 zölligen Refractor die grössten Krater auch innerhalb des Schattens noch erkannt werden; in den kleineren Fernrohren gelang das nicht. Während der zweiten Hälfte der Finsterniss zogen beständig Wolken vorüber, die die Beobachtung der Austritte aus dem Schatten sehr erschwerten. Dazu kam ein aussergewöhnlich starker Thaufall, der die Objective der transportablen Instrumente, die im Freien auf Steinpfeilern aufgestellt waren, so mit Feuchtigkeit bedeckte, dass sie häufig abgewischt werden mussten, da sonst nichts zu sehen war.

Herr Privatdocent Fewdokimow, der am Fraunhofer- 
schen Fernrohr von $84 \mathbf{m m}$ Oeffnung beobachtete, unterschied zwei Grenzlinien des Schattens und hat in einzelnen Fällen die Eintritte der Krater in beide Grenzlinien beobachtet. Im 6 zölligen Refractor konnte ich keine verschiedenen Schattengrenzen unterscheiden. Ueberhaupt erschien mir die Schattengrenze so verwaschen, dass ich die Sicherheit der Beobachtungen kaum auf $\mathbf{I}^{\mathrm{m}}$ schätzen kann.

Bei den Beobachtungen der Austritte aus dem Schatten scheinea mehrfach Verwechselungen der Mondkrater vorgekommen zu sein, wie die oft grossen Unterschiede zwischen den Resultaten der verschiedenen Beobachter anzeigen. Auffallend gross sind die Unterschiede zwischen den Beobachtungen von Tycho durch die einzelnen Beobachter, doch ist zu berücksichtigen, dass der Ein- und Austritt dieses Kraters kurz vor und nach der Maximalphase in der Nähe der Südgrenze des Schattens geschah, so dass die Beobachtung bei der Verwaschenheit der Schattengrenze sehr unsicher werden musste.

Die Beobachtungszeiten sind durchweg in Charkower Sternzeit angegeben.

$$
\text { I. Beobachter L. Struve. }
$$

Instrument: Refractor von $160 \mathrm{~mm}$ Oeffnung, Vergr. Ioo. Eintritte.

\begin{tabular}{|c|c|c|}
\hline Aristarch Mitte & $1 \eta^{\mathrm{h}}$ & $5 \cdot 2$ \\
\hline La Hire Mitte & & 9.2 \\
\hline Plato I. Rand & & 12.0 \\
\hline ॥II. Rand & & 13.2 \\
\hline Pytheas I. Rand & & I 4.4 \\
\hline$\gg \quad \Pi$. Rand & & I 4.9 \\
\hline Copernicus I. Rand & & 17.8 \\
\hline$\gg \quad$ II. Rand & & 19.9 \\
\hline Eratosthenes Mitte & & 1 $9.7:$ \\
\hline Aristoteles I. Rand & & 27.2 \\
\hline » II. Rand & & 29.2 \\
\hline Menelaus Mitte & & 31.9 \\
\hline Plinius Mitte & & $35 \cdot 3$ \\
\hline Triesnecker Mitte & & 40.0 \\
\hline Mare Crisium I. Rand & & 42.7 \\
\hline Pitatus Mitte & & $47 \cdot 7$ \\
\hline Mare Crisium II. Rand & & 50.1 \\
\hline Tycho I. Rand & I 8 & $0.0 ?$ \\
\hline Mitte & & 3.0 \\
\hline$\gg \quad$ II. Rand & & 4. 1 \\
\hline
\end{tabular}

Austritte.

\begin{tabular}{|c|c|}
\hline Tycho I. Rand & $18^{\mathrm{h}} 5 \mathrm{I}^{\mathrm{m}} \cdot 7$ \\
\hline$\gg \quad$ Mitte & 52.9 \\
\hline Pitatus I. Rand & I. 7 \\
\hline » Mitte & $3 \cdot 3$ \\
\hline III. Rand & 4.2 \\
\hline La Hire (?) Mitte & 14.2 \\
\hline Lambert (?) Mitte & 19.6 \\
\hline Timocharis I. Rand & 28.0 \\
\hline$\gg \quad$ II. Rand & 29.0 \\
\hline Aristillus Mitte & 33.2 \\
\hline $\begin{array}{cl}\text { Plato } & \text { I. Rand } \\
\gg & \text { II. Rand }\end{array}$ & $\begin{array}{l}34.7 \\
36.1\end{array}$ \\
\hline Aristoteles Mitte & 44.2 \\
\hline
\end{tabular}

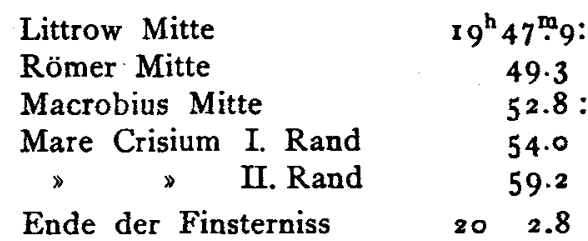

2. Beobachter Priv.-Doc. $N$. Fezdokimow.

Instr.: Fraunhofer'sches Fernrohr, $84 \mathrm{~mm}$ Oeffn., Vergr. 65.

Eintritte.

Aristarch II. Rand $\quad 7^{\mathrm{h}} 4^{\mathrm{m}} \cdot 7$ Zweite Schattengrenze

Kepler II. Rand

Archimedes II. Rand

Pallas II. Rand

Manilius Mitte

Menelaus Mitte

Plinius Mitte

Maskelyne (?) Mitte

Proclus Mitte

Coelenus Mitte

Tycho Mitte

Erste
Zweite
Erste
Zweite
Erste (?)
Erste
Zweite

Austritte.

Regiomontanus (?) Mitte $19^{\mathrm{h}}$ I $1 \frac{\mathrm{m}}{9} 9$

T. Mayer (?) Mitte

Copernicus II, Rand

19.9

Archimedes II. Rand

22.0

Aristillus Mitte

33.8

Manilius Mitte

$34 \cdot 7$

Menelaus Mitte

37.0

Plinius Mitte

Proclus (?) II. Rand

Mare Crisium I. Rand

$4 \mathrm{I.I}$

44.4

52.4

Ende der Finsterniss

53.4

$20 \quad 1.2$

3. B eobachter Stud. B. Fastremsky.

Instrument: Cometensucher, Oeffnung $96 \mathrm{~mm}$, Vergr. $3^{\circ}$. Eintritte.

Aristarch I. Rand

Grimaldi I. Rand

* II. Rand

Copernicus I. Rand

II. Rand

Manilius I. Rand

II. Rand

Menelaus I. Rand

\& II. Rand

Mare Crisium I. Rand

* II. Rand

Tycho I. Rand

Lundgreen I. Rand

Clavius I. Rand

Austritte.

Tycho I. Rand

» II. Rand

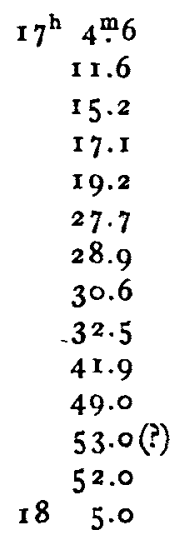

$18^{\mathrm{h}} 53^{\mathrm{m}} \cdot 2$

57.6 
Aristarch II. Rand

Copernicus II. Rand

Eratosthenes II. Rand

Petavius II. Rand

Plinius II. Rand

Theophilus II. Rand

Guttenberg (?) II. Rand

Mare Crisium II. Rand
Menelaus $\Pi$. Rand

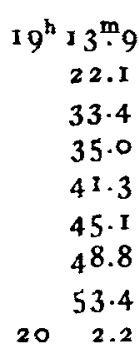

4. Beobachter Stud. A. Rasdolsky.

Instr.: Dollond'sches Fernrohr, $5^{8} \mathrm{~mm}$ Oeffnung, Vergr. 50. Eintritte.

Aristarch Mitte (?)

Grimaldi I. Rand

II. Rand

Copernicus I. Rand

Mitte

* II. Rand

Ptolemaeus I. Rand

II. Rand

Arzachel Mitte

Mare Crisium I. Rand

Schickhardt I. Rand

Charkow 1898 Juli.
Mare Crisium II. Rand

Schickhardt II. Rand

Tycho I. Rand

» Mitte

* II. Rand

Petavius I. Rand

II. Rand

Austritte

Grimaldi II. Rand

Tycho I. Rand

Aristarch Mitte

T. Mayer Mitte

Copernicus I. Rand

$\gg \quad$ Mitte

Plato I. Rand

III. Rand

Manilius Mitte

Menelaus Mitte

Plinius Mitte

Guttenberg (?) Mitte

Mare Crisium I. Rand

33.2

35.0

40.9

46.8
" $\quad$ II. Rand

Ende der Finsterniss

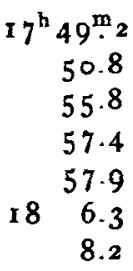

$$
\begin{aligned}
& 18^{\mathrm{h}} 5^{8^{\mathrm{m}}} \cdot 4\left(-10^{\mathrm{m}} \text { ? }\right) \\
& \text { I } 3.3\left(-10^{\mathrm{m}} \text { ? }\right) \\
& 18.1\left(-5^{\mathrm{m}} \text { ? }\right) \\
& 23.4\left(-5^{m}\right. \text { ?) } \\
& 25.6 \\
& 26.8\}\left(-5^{m ?}\right) \\
& 27.9 \\
& 36.0 \\
& 36.5 \\
& 37 \cdot 5 \\
& 41.0 \\
& 44 \cdot 5 \\
& 45-4 \\
& 46.1 \text { (?) } \\
& 59.5 \\
& 20 \quad 1.4
\end{aligned}
$$

L. Struve.

\section{Beobachtung der Mondfinsterniss 1898 Juli 3}

auf der Privatsternwarte in Landstuhl von Ph. Fauth.

Der Beginn der Finsterniss ging für die Beobachtung verloren, da der Mond im Aufgehen begriffen und vom Dunste des Horizontes verdeckt war; von $9^{\mathrm{h}} 9^{\mathrm{m}}$ bis $12^{\mathrm{k}} 15^{\mathrm{m}}$ wurden etwa 160 Notirungen über Kraterantritte des Schattens und Farben erzielt, deren bemerkenswertheste weiter unten mitgetheilt werden. Instrument: Pauly-Objectiv $178.3 \mathrm{~mm}$, Vergr. 60.5; M. E. Z., meine Taschenuhr wurde am 4. Juli mit dem Zeitsignal der Post- und Bahnstation verglichen.

$10^{\mathrm{h}} \mathrm{o}^{\mathrm{m}}$. Um das Mare Crisium und um Grimaldi (also helle Gebirgsparthien), im $\mathrm{W}$ am besten bis $25^{\circ}$ nördl. $\mathrm{Br}$., kupferglühend, innen bleigrau, gegen die Schattengrenze schmutzig graugrünlich. $-10^{\mathrm{h}} 5^{\mathrm{m}}$. Es beginnt die ganze Scheibe rosig 24 werden. - $10^{\mathrm{h}} 15^{\mathrm{m}}$. Im $\mathrm{S}$ und $\mathrm{SE}$ des Schattens noch deutlich graugrün und gelblichgrün; mit freiem Auge und im Feldstecher ist der Totaleindruck: schwach kupferbraun glühend, an den Rändern heller. - $10^{\mathrm{h}} 35^{\mathrm{m}}$. Beim Mare Crisium und Sinus Iridum herrscht kupferroth vor, gegen die Schattengrenze graugrïn, im E hellerer, härterer Ton, gelbgrünlich. $-\operatorname{II}^{\mathrm{h}} 35^{\mathrm{m}}$. Beschatteter Mondrand sehr matt, noch als röthlich erkennbar. - I I $49^{\mathrm{m}}$. Schatten scheint auszutreten. $-1^{\mathrm{h}} \mathbf{5}^{\mathbf{m}}$. Letzter Rest sicher in $19^{\circ}-20^{\circ} \mathrm{n}$. Br. verschwunden. Halbschatten als Schleier sichtbar bis Maskelyne. - ${ }^{11^{h}} 55^{\mathrm{m}}$. Umgebung des Mare Crisium leicht rauchbraun verschleiert. - Der immer matter werdende Hauch ist $12^{\text {h }} 15^{m}$, als die Beobachtung geschlossen wurde, immer noch nicht ganz verschwunden.

Der dunkle Rand wurde nie ganz unsichtbar, wenn er auch anfangs wegen der Dämmerung fast nicht zu erkennen war. Bei den Ein- und Austritten von Kratern bezieht sich die Zeitangabe (M.E.Z.) auf die Mitte der Formation.

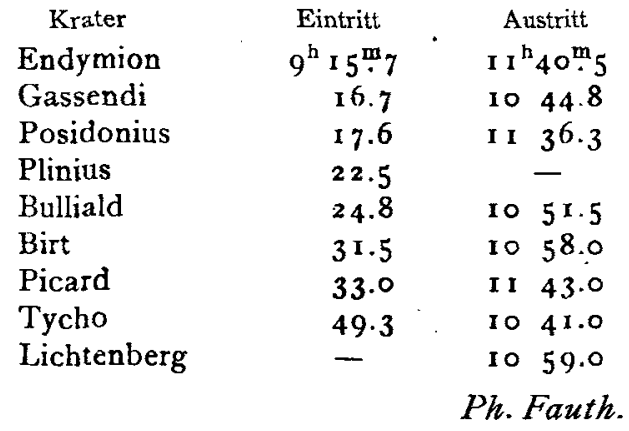

\section{Bedeckungen der Venus und von 132 Tauri 1898 Mai 22.}

Die gestrigen Bedeckungen der Venus und des Sterns $\left(5^{\text {ma }}\right)$ 1 32 Tauri wurden hier von mir am Siebenzöller und von Frau Manora am Dreizöller beobachtet. Den Moment des ersten Contacts konnten wir leider nicht vollkommen genau feststellen, weil die Nachtseite des Mondes wegen einer Dunstwolke in beiden Rohren unsichtbar war: ein Umstand, der es mir auch unmöglich machte, etwaige Lichtabnahme der Venus festzustellen. Es war $8^{\mathrm{h}} 2^{\mathrm{m}} 59^{\mathrm{s}}$ M.E.Z., 